\title{
An Approach for Power Loss Reduction in RADial Distribution Networks
}

\author{
Tan Minh PHAN $N^{1}$, Huu Ly PHAM ${ }^{2, *}$, Minh Duc Cuong NGUYEN ${ }^{3}$, \\ ${ }^{1}$ Faculty of Electrical and Electronics Engineering, Ton Duc Thang University, \\ Ho Chi Minh City 700000, Vietnam \\ ${ }^{2}$ Power System Optimization Research Group, Faculty of Electrical and Electronics Engineering, \\ Ton Duc Thang University, Ho Chi Minh City 700000, Vietnam \\ ${ }^{3}$ Faculty of Electrical-Electronics Engineering, Ly Tu Trong College of Ho Chi Minh City, \\ Ho Chi Minh City 700000, Vietnam \\ *Corresponding Author: Huu Ly PHAM (Email: phamhuuly@tdtu.edu.vn) \\ (Received: 21-Jul-2020; accepted: 13-Oct-2020; published: 31-Dec-2020) \\ DOI: http://dx.doi.org/10.25073/jaec.202044.302
}

\begin{abstract}
The loss power of distribution systems is increasingly more and more due to the increase of demand load from industrial zones and households as well as extended grids. For solving the problem, a strategy that can be regarded as a simple and effective way to allocate capacitor banks to the distribution systems. In the paper, a marine predator optimization algorithm (MPOA) is applied for determining the best size of capacitors and the most advantageous buses for the capacitors. The effectiveness of MPOA is scrutinized on two distribution systems considering the number of different integrated capacitors. The solutions found from MPOA are coped with those of recently available algorithms. Through the comparisons with previous studies, a conclusion is drawn that MPOA is a trustworthy method.
\end{abstract}

\section{Keywords}

Marine predator optimization algorithm, capacitor placement, power loss.

\section{Introduction}

In radial distribution networks, active power loss in lines accounts for a high rate of about $13 \%$ of generated power [1]. This loss is proportional to the square of the current and the resistance value of the conductor. Also, one of the challenges is to fully meet the increasing load demand with high quality and expanding lines. This leads to increased losses, low voltage, and low power quality if there is no optimal strategy for power system management and operation. From this perspective, three solutions that can deal with this problem are to use distributed generations (DG) units [2]-[4], install compensated capacitors (CC) $[5,6]$ and the feeder reconfiguration [7]. The last solution is more complex than the first two ones because an insertion of DG and $\mathrm{CC}$ into an available system offers possible profits if they are assigned positions appropriately. Optimal insertion of them into such system has attracted a huge number of attentions from researchers and engineers.

In this paper, the issue of allocating the position and determining the size of capacitors is carefully studied to give the best solution to reduce losses. Over the last years, several algorithms [8]-[20] have been introduced 
to solve the capacitor allocation problem for minimizing power loss. With various test systems, these mentioned algorithms can be divided into two groups. The first group comprising of highly effective approach (HEA) [8], particle swarm optimization with time-varying inertia weighting (PSO-TVIW) [9], ant colony method (ACM) [10], modified harmony algorithm (MHA) [11], PSO [12] and real coded genetic algorithm (RGA) [13] have been suggested for optimal location and sizing of capacitors for the 15-node network. The second group such as combined practical approach (CPA) [14], novel heuristic approach (NHA) [15], bacterial foraging optimization algorithm (BFOA) [16], flower pollination algorithm (FPA) [17], intersect mutation differential evolution (IMDE) [18], moth swarm algorithm (MSA) [19], and effective analytical technique (EAT) [20] has been introduced to seek the optimal position and rating of the shunt capacitors in 33-bus IEEE standard network in order to minimize losses and improve voltage profile. In general, the determination of position and rating of shunt capacitors in the previous papers has been solved by two ways. In the first way, candidate buses are found for assigning location of shunt capacitors, and then the calculation of the sizing of capacitors is implemented. In the second way, the optimal placement and sizing of capacitors are executed simultaneously.

This paper suggests a novel approach, named MPOA [21], to optimally discover the position and identify the size of capacitor banks in radial distribution networks. The prominent point of MPOA was proven by the experiment results when compared to other methods in terms of convergence speed and performance. As a result, the solutions of MPOA method are determined by utilizing the 15-bus and 33-bus standard distribution networks. Subsequently, this paper offers some contributions as follows:

(1) Choose the best suitable control variables for MPOA for different radial distribution networks,

(2) Analyze the weak and strong point of the structure of MPOA,
(3) Seek the best position as well as the best size for capacitors to lessen total power losses.

The organization of other parts in the paper is as follows: Section 2 describes the model establishment for the problem. MPOA is offered in Section 3. Section 4 discusses the comparison results. Finally, Section 5 presents the conclusion.

\section{Mathematic model establishment for the problem}

\subsection{Load flow calculation}

For calculating load power flow in distribution networks, one of the best algorithms called Forward-Backward sweep tool has been used [22]. The purpose of running load power flow is to identify the voltage at nodes and the branch current from a single-line diagram as given in Fig. 1.



Fig. 1: Simple distribution system.

The current $I_{j, j+1}$ flowing from node $\mathrm{j}$ to node $j+1$ is formed as Eq. (1).

$$
\left|I_{j, j+1}\right|=\left(\frac{\sqrt{P_{j, j+1}^{2}+Q_{j, j+1}^{2}}}{\left|V_{j}\right|}\right)
$$

The voltage relationship between node $\mathrm{j}$ and node $j+1$ is presented in Eq. (2) whilst real active and reactive power are given in Eq. (3) 
and Eq. (4), respectively.

$$
\begin{aligned}
V_{j}^{2} & =V_{j+1}^{2} \\
& +2 *\left(R_{j, j+1} * P_{j, j+1}+X_{j, j+1} * Q_{j, j+1}\right) \\
& -\left(R_{j, j+1}^{2}+X_{j, j+1}^{2}\right) *\left|I_{j, j+1}\right|^{2} \\
P_{j} & =P_{j+1}+P_{L, j}+R_{j, j+1} *\left|I_{j, j+1}\right|^{2} \\
Q_{j} & =Q_{j+1}+Q_{L, j}+X_{j, j+1} *\left|I_{j, j+1}\right|^{2}
\end{aligned}
$$

The loss of line connecting between node $j$ and $j+1$ can be drawn from Eqs. (3) and (4) as shown by the following equations.

$$
\begin{gathered}
P_{\operatorname{Loss}(j, j+1)}=R_{j, j+1} *\left|I_{j, j+1}\right|^{2} \\
Q_{\operatorname{Loss}(j, j+1)}=X_{j, j+1} *\left|I_{j, j+1}\right|^{2}
\end{gathered}
$$

The total $\mathrm{kW}$ loss $(\mathrm{PT})$ is reckoned by totalizing all line losses as given in Eq. (7):

$$
P_{T}=\sum_{j=1}^{N} P_{\operatorname{Loss}(j, j+1)}
$$

\subsection{Objective function of Problem}

The core goal of the problem for selecting the capacitor placement with the suitable rating is to minimize the real power losses. So, the objective function can be stated in the following formula.

$$
\text { Minimize } P_{T}=\sum_{j=1}^{N} P_{\operatorname{Loss}(j, j+1)}
$$

\subsection{Constraints}

Such objective function is governed by constrain conditions as follows

Voltage requirement: The voltage magnitude of every node should be restricted within the following range:

$$
V_{\min } \leq V_{j} \leq V_{\max } ; j=1, \ldots, N
$$

Injected reactive power requirement: Total compensated reactive power must be limited by

$$
\sum Q_{c} \leq \sum_{j}^{N} Q_{L, j} ; j=1, \ldots, N
$$

\section{Marine predators optimization algorithm (MPOA)}

MPOA was developed in the early 2020. It is formulated based on hunting behavior of predators in marine ecosystems [21]. In MPOA, Brownian and Lévy flights distributions were used for the widespread foraging strategy. In which, Brownian distribution is suitable to search solutions in local domains whilst Lévy flights distribution is useful to search solutions in large domains. In [21], the authors have proven the performance of MPOA through solving some traditional benchmark test functions and engineering design problems. In recent times, MPOA has been successfully employed for forecasting confirmed cases of COVID-19 in Italy, USA, Iran and Korea [23]. The process of updating new solutions of MPOA is characterized by three stages corresponding to the current iterations with different strategies. The steps of the MPOA are explained as follows:

The first stage: This stage is carried out when the iteration is run from 1 to one-third of the maximum number iterations $\left(\operatorname{Max}_{\text {iter }}\right)$. In such stage, newly updated solutions are created by

$$
\begin{gathered}
P_{i}=P_{i}+\alpha * \beta \otimes \operatorname{delta}_{i} ; i=1 \ldots S_{\text {pop }} \\
\operatorname{delta}_{i}=\varepsilon \otimes\left(P_{\text {best }}-\varepsilon \otimes P_{i}\right)
\end{gathered}
$$

The second stage: This stage is conducted when the iteration is higher than one-third of Maxiter and equal or smaller than two-third of $M_{a x} x_{i t e r}$. In such stage, two strategies are suggested for both exploration and exploitation issues. The population size $\left(S_{p o p}\right)$ should be therefore divided into two equal groups to be in charge of the mentioned task. The approach for the mission of the first group is suggested by

$$
\begin{aligned}
& P_{i}=P_{i}+\alpha * \beta \otimes \operatorname{delta}_{i} ; i=1 \ldots \frac{S_{p o p}}{2} \\
& \operatorname{delta}_{i}=\phi \otimes\left(P_{\text {best }}-\phi \otimes P_{i}\right)
\end{aligned}
$$




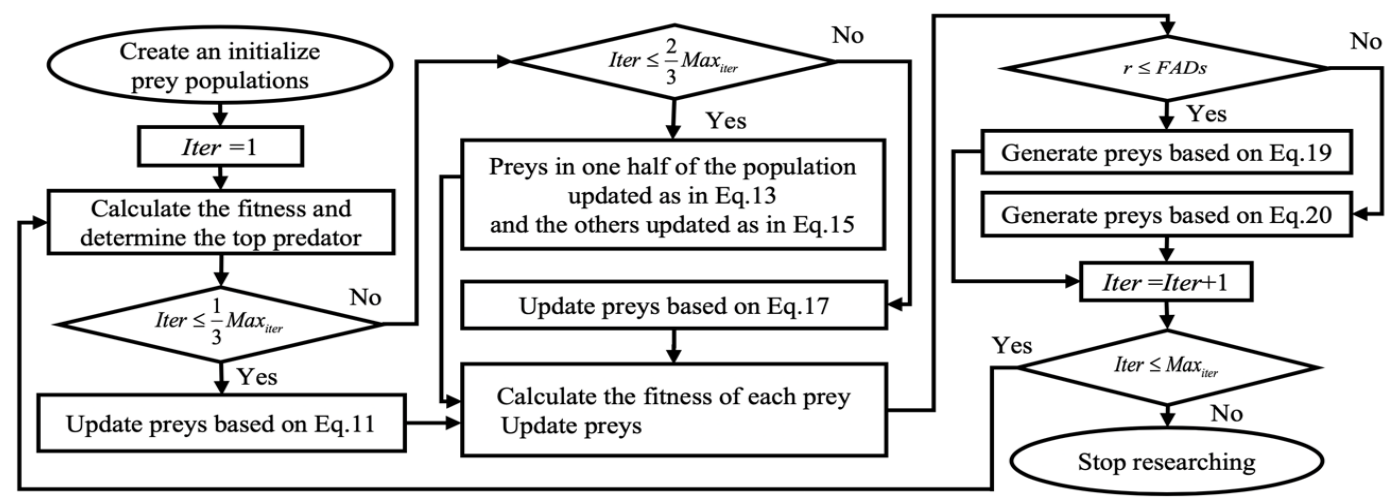

Fig. 2: Simple distribution system.

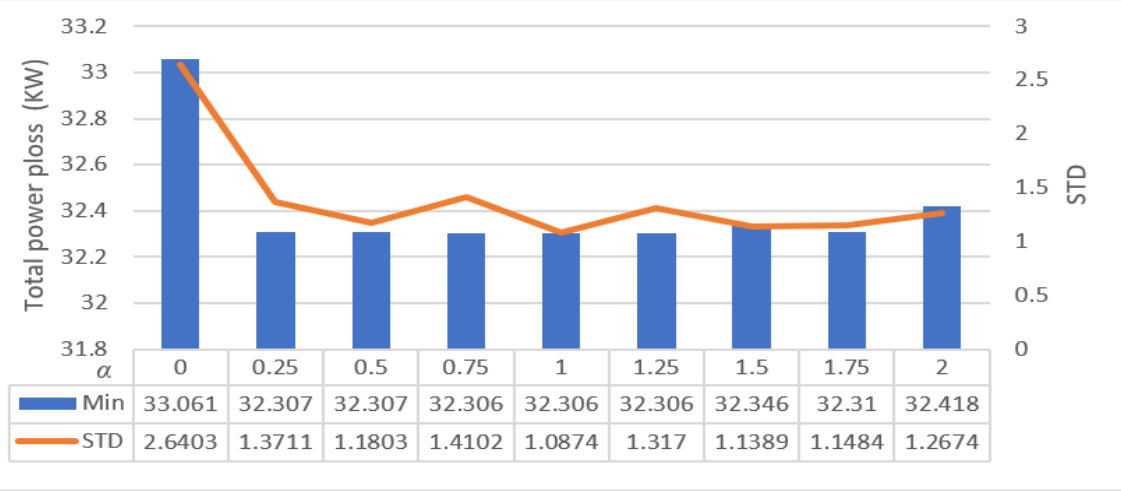

Fig. 3: The obtained results from changing of $\alpha$.

\begin{tabular}{|c|c|c|c|c|c|c|c|c|c|}
\hline$\alpha$ & 0 & 0.25 & 0.5 & 0.75 & 1 & 1.25 & 1.5 & 1.75 & 2 \\
\hline Min & 33.061 & 32.307 & 32.307 & 32.306 & 32.306 & 32.306 & 32.346 & 32.310 & 32.418 \\
\hline Aver & 36.860 & 34.112 & 33.814 & 34.073 & 34.051 & 34.122 & 34.318 & 33.987 & 34.335 \\
\hline Max & 47.594 & 38.209 & 36.642 & 37.821 & 36.272 & 37.046 & 36.901 & 36.492 & 36.900 \\
\hline STD & 2.640 & 1.371 & 1.180 & 1.410 & 1.087 & 1.317 & 1.139 & 1.148 & 1.267 \\
\hline
\end{tabular}

Tab. 1: The results from the investigation of $\alpha$.

The approach for the work of another is pro- In this stage, new solutions are generated by posed by

$$
\begin{aligned}
& P_{i}=P_{b e s t} \\
& +\alpha *\left(1-\frac{\text { Iter }}{\text { Max }_{i t e r}}\right)^{\left(2 * \frac{\text { Iter }}{\text { Maxiter }}\right)} \otimes \operatorname{delta} 2_{i} ; \\
& i=\frac{S_{p o p}}{2} \ldots S_{p o p} \\
& \operatorname{delta} 2_{i}=\phi \otimes\left(\phi \otimes P_{\text {best }}-P_{i}\right) \\
& P_{i}=P_{\text {best }} \\
& +\alpha *\left(1-\frac{\text { Iter }}{\text { Max }}\right)^{\left(2 * \frac{\text { Iter }}{\text { Maxiter }}\right)} \otimes \operatorname{delta3_{i}} ; \\
& i=1 \ldots S_{\text {pop }}
\end{aligned}
$$

The last stage: This stage is happened when the iteration is higher than two-third of Maxiter.

$$
\operatorname{delta} 3_{i}=\phi \otimes\left(\phi \otimes P_{\text {best }}-P_{i}\right)
$$


After each stage, solutions will be created by a comparison criterion as the following models

$$
\begin{aligned}
& P_{i}=P_{i} \\
& +\left(1-\frac{\text { Iter }}{M a x_{i t e r}}\right)^{\left(2 * \frac{\text { Iter }}{M a x_{i t e r}}\right)} \\
& *\left[P_{\min }+\omega \otimes\left(P_{\max }-P_{\min }\right)\right] \otimes \nu \\
& \text { if } r \leq F A D s \\
& P_{i}=P_{i}+[F A D s(1-r)+r]\left(P_{r 1}-P_{r 2}\right) \\
& \text { if } r>F A D s
\end{aligned}
$$

Finally, a comparison between each newly updated solution and old solution is executed to hold a better one and abandon another one. A detailed description of the solution search process of MPOA method is shown by the following flow chart as in Fig. 2.

\section{Comparison and discussion}

In this portion, the positive power of MPOA against power losses of the considered power system, total capacity of capacitors and the placement of capacitors are carefully analyzed. Distribution network of 15 and 33 buses has been utilized with different sub-cases. Fifty independent trial runs have been performed for three cases of each test system. The work for the encoding of MPOA is coded on MATLAB and run on a personal $\mathrm{PC}$.

\subsection{Analysis of MPOA parameters to test systems}

Analyzing and adjusting the control variables of each method do not lack in optimization problems because these variables can give the best result for this problem but the worst result for different problems. In MPOA method, two control variables such as the predetermined number $(\alpha)$ and FADs are investigated for adding two capacitors into the distribution system of 15 buses. In this work, 10 preys have been selected over 50 independent runs with $M a x_{i t e r}=10$. For determining $\alpha$, we set $F A D s=0.5$ and the obtained results from the simulation are presented in Tab. 1 and plotted in Fig. 3. In Fig. 3, $\alpha$ is adjusted from 0 to 2 with a size step of 0.25 . With each value of $\alpha$, the power loss and the standard deviation (STD) are determined. The comer is used to access the power of the method meanwhile the latter is employed to analyze the robustness. Observing such Fig. 3 indicates that the power loss of $32.306 \mathrm{~kW}$ and STD of 1.0874 are the best values tantamount to $\alpha=1.0$.

For determining the best value of $F A D s$, the value of $\alpha$ is set to 1 while $F A D s$ is set to from 0 to 1 . If $F A D s$ is smaller than 1, Eq. (19) is applied to update new solutions. Otherwise, Eq. (20) is employed. Following the rule, the results from the setting of FADs is provided in Tab. 2 and displayed in Fig. 4.

Figure 4 shows that when FADs equals 0, the best loss of $32.306 \mathrm{~kW}$ and STD of 0.997 are the best values for this test system with two capacitors. From the above survey results, $\alpha=1$ and $F A D s=0$ are applied to evaluate the ability of MPOA method for different cases.

\subsection{The 15-bus network}

The load demand of 15-bus network is 1752 . 07 $\mathrm{kVA}$ and the power loss in the transmission line of the system is $61.8 \mathrm{~kW}[8]$. The replete performance of each considered system is directly influenced by connecting the number of capacitors that should be carefully investigated to achieve the best optimal solution. Table 3 provides a comparison of results achieved by the suggested MPOA and other techniques for three Cases, Case 1 with two capacitors, Case 2 with three capacitors, and Case 3 with five capacitors. Besides, FADs and $\alpha$ are set as in Section 4.1, $S_{\text {pop }}$ is set to be 10 and $\operatorname{Max}_{\text {iter }}$ is set to 10,30 , and 40 for Cases 1, 2, and 3, respectively.

For Case 1, HEA [8] and PSO-TVIW [9] specify the same positions of two capacitors at nodes 3 and 6 while the power loss of two methods is no significant difference with 32.6 and 32.7 , respectively. Similarly, the placements of the two 


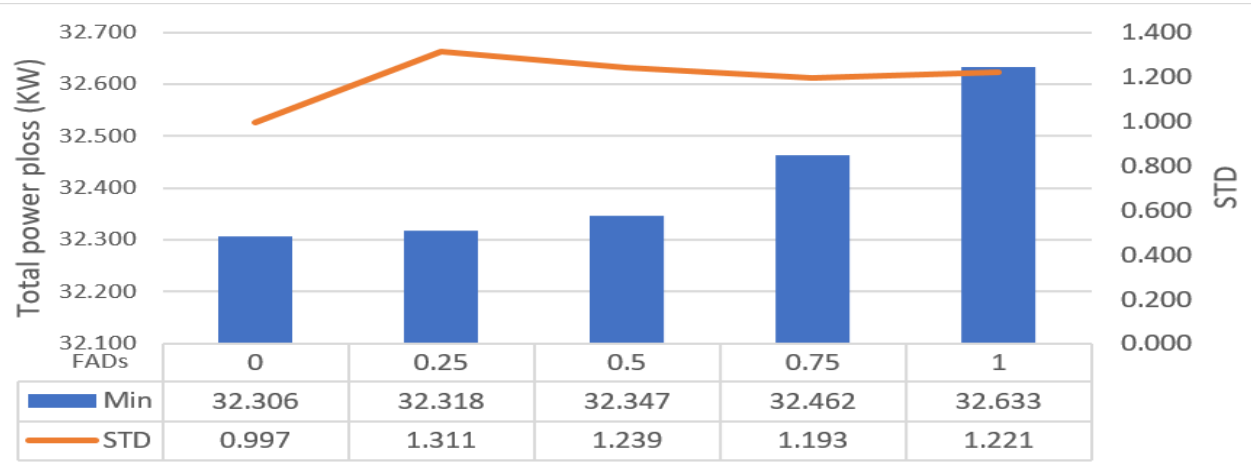

Fig. 4: Simple distribution system.

\begin{tabular}{|c|c|c|c|c|c|}
\hline FADs & 0 & 0.25 & 0.5 & 0.75 & 1 \\
\hline Min & 32.306 & 32.318 & 32.347 & 32.462 & 32.633 \\
\hline Aver & 33.875 & 34.207 & 34.313 & 34.180 & 34.484 \\
\hline Max & 36.417 & 37.034 & 36.703 & 36.935 & 37.175 \\
\hline STD & 0.997 & 1.311 & 1.239 & 1.193 & 1.221 \\
\hline
\end{tabular}

Tab. 2: The results from the investigation of FADs.

\begin{tabular}{|c|c|c|c|c|c|}
\hline \multirow{3}{*}{ Study case } & Methods & Capacitor & Size & Total size & Total loss \\
& & site (node) & $(\mathrm{kVAr})$ & $(\mathrm{kVAr})$ & $(\mathrm{kW})$ \\
\hline \multirow{4}{*}{1} & HEA [8] & 3,6 & 805,388 & 1,193 & 32.6 \\
\cline { 2 - 6 } & PSO-TVIW [9] & 3,6 & 871,321 & 1,192 & 32.7 \\
\cline { 2 - 6 } & ACM [10] & 4,6 & 630,410 & 1,040 & 36.81 \\
\cline { 2 - 6 } & MPOA & 4,6 & 705,435 & 1,140 & 32.3063 \\
\hline \multirow{3}{*}{2} & MHA [11] & $6,11,15$ & $350,300,300$ & 950 & 31.12 \\
\cline { 2 - 6 } & MPOA & $4,6,11$ & $491,406,298$ & 1,195 & 30.34 \\
\hline \multirow{3}{*}{3} & PSO [12] & $4,6,7,11,15$ & $274,193,143,267,143$ & 1,020 & 30.5522 \\
\cline { 2 - 6 } & RGA [13] & $4,6,7,11,15$ & $274,193,143,267,143$ & 1,020 & 30.5522 \\
\cline { 2 - 6 } & MPOA & $2,4,6,7,12$ & $198,473,241,147,215$ & 1,274 & 29.9128 \\
\hline
\end{tabular}

Tab. 3: MPOA results for three cases compared with other techniques in 15-Bus.

capacitors identified by ACM [10] and MPOA are nodes 4 and 6 ; however, the power loss of MPOA is the best. For Case 2, MHA [11] and MPOA can reduce the loss more effective than the case of the base network without reactive compensation. This value proves that MPOA is more impressive than MHA [11] with the power loss of $30.34 \mathrm{~kW}$ compared to $31.12 \mathrm{~kW}$. For Case 3 , results from PSO [12] and RGA [13] in terms of capacitor placements and sizes, total compensated power and total power loss are exactly the same. Comparing with two mentioned methods can recognize that the power loss of $29.9128 \mathrm{~kW}$ from MPOA is better. From here, we can evaluate that MPOA is stronger than other methods for three study cases. For the investigation of voltage profile improvement, Fig. 5 is drawn to display the change of the voltage of nodes in 15bus system for different cases.

\subsection{The 33-bus network}

In this section, the network with 33 buses is used to inspect the performance of MPOA by analyzing three cases with one capacitor, two capac- 
itors and three capacitors. These parameters of MPOA are also set as follows: $F A D s=0$; $\alpha=1 ; S_{p o p}=10$, and $M a x_{i t e r}=30,30$, and 50 for three cases, respectively. The results collected from MPOA are compared to those of other tools as shown in Tab. 4. For Case 1, three methods including CPA [14], NHA [15], and MPOA find the same position at node 30 . The power loss of CPA [14] and NHA [15] are approximately equal to power loss, that of MPOA is a little smaller. For Case 2, nodes 12 and 30 are regarded as the most suitable positions for capacitors found by CPA [14] and MPOA while those of NHA [15] are nodes 13 and 30 but the power loss of MPOA is the best among the three methods. For Case 3, total loss from MPOA are compared to that from the seven other methods such as CPA [14], NHA [15], BFOA [16], FPA [17], IMDE [18], MSA [19], and EAT [20]. As seeing the second column in the table, nodes 12 , 24, and 30 are found by CPA [14], MSA [19], and MPOA, nodes 18, 30, and 33 are discovered by BFOA [16] and IMDE [18], nodes 13, 24, and 30 are detected by FPA [17] and EAT [20] while those of NHA [15] are nodes 13, 25, and 30 , respectively. When seeing the last column, three methods including CPA [14], MSA [19], and MPOA can reach better loss than other ones corresponding to nodes 12,24 , and 30. Clearly, three mentioned nodes are the best suitable positions for inserting the compensated capacitors. In relation to the power loss level, MSA is the best one, MPOA is the second best one while BFOA is the worst one. From here, it can be implied that MPOA is a potential method. For inspecting the improvement of the voltage of nodes in the 33-bus system before and after installing capacitors, Fig. 6 is plotted. From such Figure, it can be seen that when the system is integrated with capacitors, the voltage of nodes is significantly enhanced. However, if we conduct a comparison of three compensated cases, Case 3 has better voltage profile than others.

\section{Conclusions}

In this paper, we have used the MPOA method for an important task to be reduced the active power loss in distribution systems. The mission of MPOA is to seek the proper location and sizing of fixed capacitor banks. The method behavior is tested on two distribution networks such as 15-bus and 33-bus networks. For proving the performance of MPOA, we carry out to collect its results of different cases in the two systems. Then, a result comparison between MPOA and other methods available in recent literature has been implemented. As a result, MPOA can provide a very reliable solution for all cases of the two systems.

\section{Nomenclature}

\begin{tabular}{|c|c|}
\hline$\beta, r, \omega, v$ & $\begin{array}{l}\text { Number randomly selected } \\
\text { in }[0,1]\end{array}$ \\
\hline$\varepsilon$ & $\begin{array}{l}\text { Brownian distribution based } \\
\text { random number vector }\end{array}$ \\
\hline$F A D s$ & Fish aggregating devices \\
\hline Iter & Current iteration \\
\hline$N$ & Number of buses \\
\hline$P_{j, j+1}$ & $\begin{array}{l}\text { Real power of the branch } \\
\text { connecting the buses } \\
j \text { and } j+1 \text {. }\end{array}$ \\
\hline $\operatorname{Pr} 1, \operatorname{Pr} 2$ & Two random solutions \\
\hline$P_{\text {best }}$ & $\begin{array}{l}\text { The best solution called } \\
\text { the top predator }\end{array}$ \\
\hline$P_{i}$ & The ith solution \\
\hline$P_{\min }, P_{\max }$ & $\begin{array}{l}\text { The minimum and maximum } \\
\text { bounds of the solution }\end{array}$ \\
\hline$P_{L j}, Q_{L j}$ & $\begin{array}{l}\text { Active and reactive power } \\
\text { of load at the bus } j\end{array}$ \\
\hline$Q_{j, j+1}$ & $\begin{array}{l}\text { Reactive power of the branch } \\
\text { connecting the buses } \\
j \text { and } j+1\end{array}$ \\
\hline$Q_{\min }, Q_{\max }$ & $\begin{array}{l}\text { The minimum and maximum } \\
\text { rated power of capacitor }\end{array}$ \\
\hline$Q_{c}$ & Compensated reactive power \\
\hline$R_{j, j+1}$ & $\begin{array}{l}\text { Resistance of the branch } \\
\text { connecting the buses } j \\
\text { and } j+1\end{array}$ \\
\hline$S_{\text {pop }}$ & Population size \\
\hline$\phi$ & Lévy flights distribution \\
\hline$V_{j}$ & $\begin{array}{l}\text { Magnitude of voltage at } \\
\text { the bus } j\end{array}$ \\
\hline$V_{\min }, V_{\max }$ & $\begin{array}{l}\text { The minimum and maximum } \\
\text { voltages at the bus } j\end{array}$ \\
\hline$X_{j, j+1}$ & $\begin{array}{l}\text { Reactance of the branch } \\
\text { connecting the buses } \\
j \text { and } j+1\end{array}$ \\
\hline
\end{tabular}




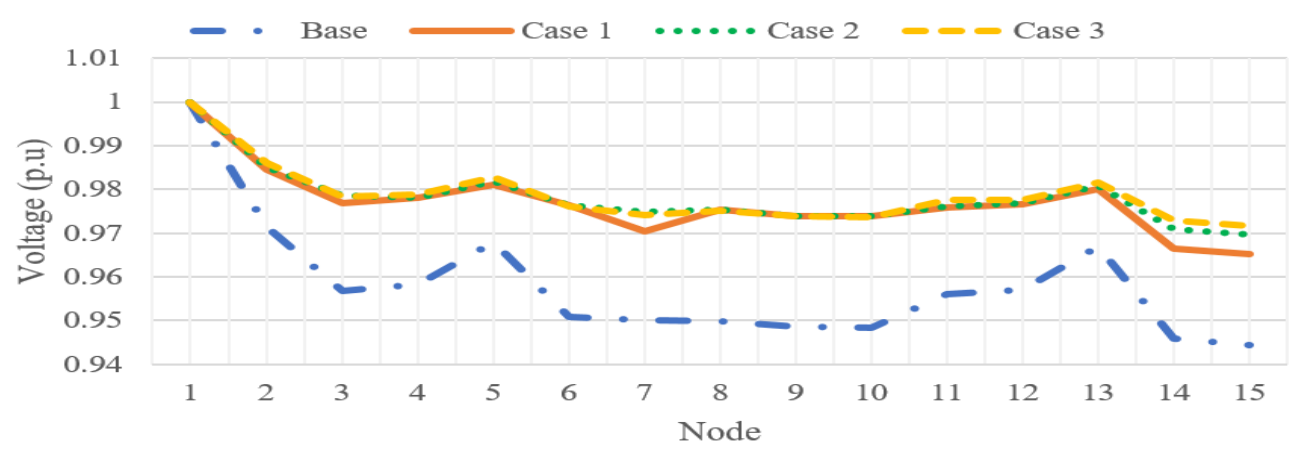

Fig. 5: The voltage of nodes in the 15-bus network for different study cases.

\begin{tabular}{|c|c|c|c|c|c|}
\hline \multirow{3}{*}{ Study case } & Methods & Capacitor & Size & Total size & Total loss \\
& & site (node) & $(\mathrm{kVAr})$ & $(\mathrm{kVAr})$ & $(\mathrm{kW})$ \\
\hline \multirow{3}{*}{1} & CPA [14] & 30 & 1250 & 1,250 & 151.52 \\
\cline { 2 - 6 } & NHA [15] & 30 & 1190 & 1,190 & 151.55 \\
\cline { 2 - 6 } & MPOA & 30 & 1258 & 1,258 & 151.38 \\
\hline \multirow{5}{*}{2} & CPA [14] & 12,30 & 440,1100 & 1,540 & 142.03 \\
\cline { 2 - 6 } & NHA [15] & 13,30 & 405,1052 & 1,457 & 141.9 \\
\cline { 2 - 6 } & MPOA & 12,30 & 1063,465 & 1,528 & 141.85 \\
\hline \multirow{5}{*}{3} & CPA [14] & $12,24,30$ & $500,500,1000$ & 2,000 & 138.61 \\
\cline { 2 - 6 } & NHA [15] & $13,25,30$ & $383,386,1000$ & 1,769 & 138.65 \\
\cline { 2 - 6 } & BFOA [16] & $18,30,33$ & $249.6,820.6,277.3$ & $1,347.5$ & 144.04 \\
\cline { 2 - 6 } & FPA [17] & $13,24,30$ & $450,450,900$ & 1,800 & 139.075 \\
\cline { 2 - 6 } & IMDE [18] & $18,30,33$ & $350,820,277$ & 1,447 & 144.04 \\
\cline { 2 - 6 } & MSA [19] & $12,24,30$ & $450,600,900$ & 1,950 & 137.23 \\
\cline { 2 - 6 } & EAT [20] & $13,24,30$ & $359,520,1016$ & 1,895 & 138.37 \\
\cline { 2 - 6 } & MPOA & $12,24,30$ & $449,496,1016$ & 1,961 & 138.34 \\
\hline
\end{tabular}

Tab. 4: MPOA results for three cases comparable with other techniques in 33- Bus.

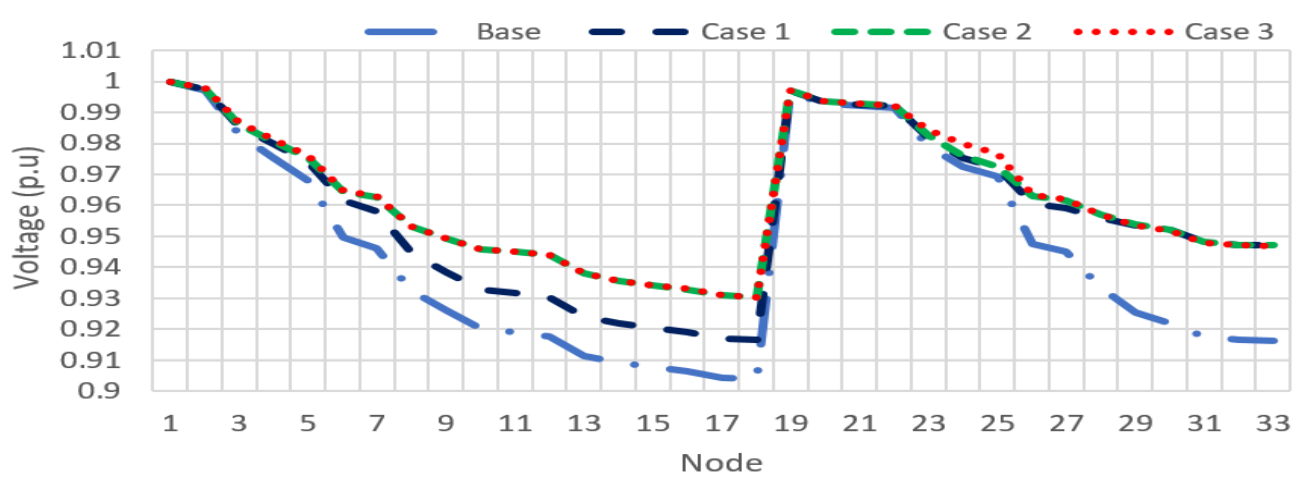

Fig. 6: The voltage of nodes in the 33-bus network for different study cases.

\section{References}

placement in a radial distribution system

[1] Rao, R. S., Narasimham, S. V. L., \& Ramalingaraju, M. (2011). Optimal capacitor 
using plant growth simulation algorithm. International journal of electrical power \& energy systems, 33(5), 1133-1139.

[2] Cabana, K., Candelo, J., Castillo, R., \& De-la-Hoz-Franco, E. (2019). Voltage sensitivity analysis to determine the optimal integration of distributed generation in distribution systems.

[3] Sridhar, J. P., \& Prakash, R. (2019). Multiobjective whale optimization based minimization of loss, maximization of voltage stability considering cost of DG for optimal sizing and placement of DG. International Journal of Electrical and Computer Engineering (IJECE), 9(2), 835-839.

[4] Ang, S., Leeton, U., Chayakulkeeree, K., \& Kulworawanichpong, T. Sine Cosine Algorithm for Optimal Placement and Sizing of Distributed Generation in Radial Distribution Network.

[5] Elsayed, A. M., Mishref, M. M., \& Farrag, S. M. (2019). Optimal allocation and control of fixed and switched capacitor banks on distribution systems using grasshopper optimisation algorithm with power loss sensitivity and rough set theory. IET Generation, Transmission \& Distribution, 13(17), 3863-3878.

[6] Auchariyamet, S., \& Sirisumrannukul, S. (2009). Optimal capacitor placement in unbalanced loading distribution system with nonlinear loads by adaptive particle swarm technique. GREATER MEKONG SUBREGION ACADEMIC AND RESEARCH NETWORK, 91.

[7] Rugthaicharoencheep, N., \& Sirisumrannukul, S. (2009). Feeder reconfiguration for loss reduction in distribution system with distributed generators by tabu search. GMSARN International Journal, 3, 47-54.

[8] Haque, M. H. (1999). Capacitor placement in radial distribution systems for loss reduction. IEE Proceedings-Generation, Transmission and Distribution, 146(5), 501-505.
[9] Prakash, K., \& Sydulu, M. (2007, June). Particle swarm optimization based capacitor placement on radial distribution systems. In 2007 IEEE power engineering society general meeting (pp. 1-5). IEEE.

[10] Abou El-Ela, A. A., Kinawy, A. M., Mouwafi, M. T., \& El-Sehiemy, R. A. (2015, September). Optimal sitting and sizing of capacitors for voltage enhancement of distribution systems. In 2015 50th International Universities Power Engineering Conference (UPEC) (pp. 1-6). IEEE.

[11] Ali, E. S., Abd Elazim, S. M., \& Abdelaziz, A. Y. (2016). Improved harmony algorithm and power loss index for optimal locations and sizing of capacitors in radial distribution systems. International Journal of Electrical Power \& Energy Systems, 80, 252263.

[12] Reddy, M. D., \& Reddy, V. V. (2008). Capacitor placement using fuzzy and particle swarm optimization method for maximum annual savings. ARPN Journal of Engineering and Applied Sciences, 3(3), 25-30.

[13] Reddy, M. D. (2008). Optimal capacitor placement using fuzzy and real coded genetic algorithm for maximum savings.

[14] Hung, D. Q., Mithulananthan, N., \& Bansal, R. C. (2015). A combined practical approach for distribution system loss reduction. International Journal of Ambient Energy, 36(3), 123-131.

[15] Bayat, A., \& Bagheri, A. (2019). Optimal active and reactive power allocation in distribution networks using a novel heuristic approach. Applied Energy, 233, 71-85.

[16] Kowsalya, M. I. A. M. (2014, January). Optimal Distributed Generation and capacitor placement in power distribution networks for power loss minimization. In 2014 International Conference on Advances in Electrical Engineering (ICAEE) (pp. 1-6). IEEE.

[17] Prakash, D. B., \& Lakshminarayana, C. (2017). Optimal siting of capacitors in radial distribution network using whale optimization algorithm. Alexandria Engineering Journal, 56(4), 499-509. 
[18] Khodabakhshian, A., \& Andishgar, M. H. (2016). Simultaneous placement and sizing of DGs and shunt capacitors in distribution systems by using IMDE algorithm. International Journal of Electrical Power \& Energy Systems, 82, 599-607.

[19] Mohamed, E. A., \& Mitani, Y. (2018). MSA for optimal reconfiguration and capacitor allocation in radial/ring distribution networks. IJIMAI, 5(1), 107-122.

[20] Kamel, S., Amin, A., Selim, A., \& Ahmed, M. H. (2019, September). Optimal Placement of DG and Capacitor in Radial Distribution Systems Considering Load Variation. In 2019 International Conference on Computer, Control, Electrical, and Electronics Engineering (ICCCEEE) (pp. 1-6). IEEE.

[21] Faramarzi, A., Heidarinejad, M., Mirjalili, S., \& Gandomi, A. H. (2020). Marine predators algorithm: A nature-inspired Metaheuristic. Expert Systems with Applications, 113377.

[22] Quintero-Duran, M., Candelo-Becerra, J. E., \& Soto-Ortiz, J. D. (2019). A modified backward/forward sweep-based method for reconfiguration of unbalanced distribution networks. International Journal of Electrical and Computer Engineering, 9(1), 85101.

[23] Al-Qaness, M. A., Ewees, A. A., Fan, H., Abualigah, L., \& Abd Elaziz, M. (2020). Marine Predators Algorithm for Forecasting Confirmed Cases of COVID-19 in Italy, USA, Iran and Korea. International Journal of Environmental Research and Public Health, 17(10), 3520.

\section{About Authors}

Tan Minh PHAN received his B.Eng degrees in Electrical Engineering from University of Ton Duc Thang. Now, he is studying at department of electrical and electronics engineering, Ton Duc Thang University. His research interests include optimization of power system, power system operation and control and Renewable Energy.

Huu Ly PHAM was born in Dong Thap, Vietnam. He received the B.Sc. and MSc degree in Electrical Engineering from Ho Chi Minh City University of Technology, Vietnam in 2004 and 2008, respectively. Currently, he works at Faculty of electrical \& electronics engineering, Ton Duc Thang University. His interests include optimization of power system, power system operation and control and Renewable Energy.

Minh Duc Cuong NGUYEN received his B. Eng and M.Eng degree in Electrical Engineering from University of Technical education HCM City. Now, he is a researcher of Faculty of electrical \& electronics engineering, Ly Tu Trong College in HCM city. His research interests include optimization of power system, power system operation and control and Renewable Energy. 